\title{
PREFERENTIAL SORPTION VERSUS PREFERENTIAL PERMEABILITY IN PERVAPORATION*
}

\author{
M.H.V. MULDER, T. FRANKEN and C.A. SMOLDERS
}

Department of Chemical Technology, Twente University of Technology, P.O. Box 217, 7500 AE Enschede (The Netherlands)

(Received November 2, 1983; accepted February 2, 1984)

\section{Summary}

Transport of liquids by pervaporation takes place by a solution-diffusion mechanism. In order to investigate the "solution part" of this transport model, preferential sorption has been compared with preferential permeability. Sorption equilibria and pervaporation experiments for the systems water-ethanol-cellulose acetate, water-ethanol-polyacrylonitrile and water-ethanol-polysulfone have been investigated. Theoretical values of preferential sorption have been derived from Flory-Huggins thermodynamics, extended with concentration dependent interaction parameters. These calculated sorption values show a reasonable agreement with experimental values. The large difference in molar volumes between water and ethanol determines the preferential sorption of water in these systems to a great extent, and this effect increases with decreasing swelling value. Comparison of preferential sorption experiments with pervaporation experiments indicates that, apart from the effect of differences in diffusivity for the permeating components, preferential sorption contributes to a major extent to selective transport

\section{Introduction}

In most membrane processes transport of molecules takes place in the direction of decreasing chemical potential. In pervaporation, the driving force for transport is the concentration difference across the membrane. The transport process can be divided into three steps, (i) sorption into the membrane at the upstream side, (ii) diffusion through the membrane, and (iii) desorption into a vapour phase at the downstream side. The separation mechanism of pervaporation is a solution-diffusion mechanism [1-4], i.e., the permeation rate is a function of solubility and diffusivity. Solubility is a thermodynamic property and diffusivity is a kinetic property, both affecting selectivity. In the case of a liquid mixture, separation is obtained because the membrane has the ability to transport one component more readily than the other even if the driving forces are equal. Hence, prediction of selectivity is

\footnotetext{
*Paper presented at the 4th Symposium on Synthetic Membranes in Science and Industry, Tübingen, F.R.G., September 6-9, 1983.
} 
often difficult because there will be in general a coupling of fluxes, i.e., the permeation rate of one component can be changed by the presence and movement of the other component. In a recent article [4] a solution-diffusion model has been developed for the permeation of a liquid mixture through a polymeric membrane taking into account coupling of fluxes.

The objective of this paper is to investigate the thermodynamic aspects of the membrane separation process, by comparing preferential sorption of a water-ethanol mixture by a polymeric membrane with preferential permeability through that membrane.

Aptel [5] showed that for systems with polyvinylpyrolidone-polytetrafluoroethylene as membrane material and various binary liquid mixtures, the component that was sorbed preferentially was also transported preferentially. Even the occurrence of an inversion in selectivity was in agreement with their sorption experiments.

Our investigation can be divided into two parts:

(a) the thermodynamics of preferential sorption;

(b) a comparison of preferential sorption versus preferential permeability.

Preferential or selective sorption is given by the difference in composition of a binary liquid mixture inside the polymeric membrane, and outside in the liquid feed mixture. Theoretical values on preferential sorption have been derived from Flory-Huggins thermodynamics [6] using concentration dependent interaction parameters. To improve the agreement between experimental and theoretical data on preferential sorption Pouchly $[7,8]$ introduced a second-order interaction parameter, the ternary parameter $g_{T}$. Another way of describing second-order effects is by taking the interaction parameters to be concentration dependent. In this paper we will follow the latter approach.

Experimental data on preferential sorption have been obtained by separating the sorbed liquid quantitatively from the membrane phase by a distillation technique. These experimental data will be compared with the theoretical values. The following polymers have been studied: cellulose acetate (CA), polyacrylonitrile (PAN) and polysulfone (PSf), while water-ethanol was used as the liquid mixture. Except for equilibrium sorption measurements, pervaporation experiments have also been performed. The selectivity in pervaporation will be compared with the preferential sorption data, and the results will be discussed in terms of the solution-diffusion mechanism.

\section{Theory}

The equilibrium between a polymeric membrane (index 3 ) and a binary mixture of nonsolvents (indices 1 and 2) can be considered as an osmotic equilibrium. Preferential sorption occurs when the composition of the binary liquid mixture inside the polymer is different from that outside, in the liquid feed mixture. The index 1 is given to the component that is sorbed preferentially by the polymer. If we denote the concentration of a component of a binary liquid mixture in the polymeric phase by 
$u_{i}=\frac{\phi_{i}}{\phi_{1}+\phi_{2}}=\frac{\phi_{i}}{1-\phi_{3}} \quad i=1,2$

and the concentration (volume fraction) in the liquid phase by $v_{l}$ then the preferential sorption $\epsilon$ is given by [7]

$\epsilon=u_{1}-v_{1}=v_{2}-u_{2}$

The condition for equilibrium between the two phases, the binary liquid phase and the ternary polymer phase, are expressed by equality of the chemical potentials in the two phases. The polymer-free phase is denoted with the superscript $o$ and the ternary (membrane) phase with the superscript $m$. At equilibrium we have:

$\Delta \mu_{1}^{o}=\Delta \mu_{1}^{\mathfrak{m}}+\Pi V_{1}$

$\Delta \mu_{2}^{\mathbf{0}}=\Delta \mu_{2}^{\mathrm{m}}+\Pi V_{2}$

The chemical potentials can be obtained from Flory-Huggins thermodynamics [6]. The Gibbs free energy of mixing of a ternary system is given by

$$
\begin{aligned}
\frac{\Delta G_{\mathrm{m} 1 \mathbf{x}}}{R T}= & n_{1} \ln \phi_{1}+n_{2} \ln \phi_{2}+n_{3} \ln \phi_{3}+g_{12}\left(u_{2}\right) n_{1} \phi_{2}+ \\
& g_{13}\left(u_{2}, \phi_{3}\right) n_{1} \phi_{3}+g_{23}\left(u_{1}, \phi_{3}\right) n_{2} \phi_{3}
\end{aligned}
$$

The indices 1 and 2 again refer to the nonsolvents, and index 3 to the polymer; $n_{t}$ and $\phi_{i}$ are the mole fraction and volume fraction of component $i$, respectively. The binary interaction parameters $g_{12}, g_{13}$ and $g_{23}$ are assumed to be concentration-dependent. In case these parameters are independent of concentration, the $g$ parameters equal the $\chi$ parameters. (In the original FloryHuggins theory the $\chi$ parameters are concentration-independent [6].) Differentiation of eqn. (5) to $n_{1}$ and $n_{2}$, respectively, yields the following equations for the chemical potentials of components 1 and 2 in the polymer phase

$$
\begin{aligned}
\frac{\Delta \mu_{1}^{\mathrm{m}}}{R T}= & \ln \phi_{1}+\phi_{2}\left(1-\frac{V_{1}}{V_{2}}\right)+\phi_{3}\left(1-\frac{V_{1}}{V_{3}}\right)+\left(g_{12} \phi_{2}+g_{13} \phi_{3}\right)\left(\phi_{2}+\phi_{3}\right) \\
& -u_{1} u_{2} \phi_{2} \frac{\partial g_{12}}{\partial u_{2}}-g_{23} \frac{V_{1}}{V_{2}} \phi_{2} \phi_{3}-u_{1} u_{2} \phi_{3} \frac{\partial g_{13}}{\partial u_{2}}-\phi_{1} \phi_{3}^{2} \frac{\partial g_{13}}{\partial \phi_{3}} \\
& +\frac{V_{1}}{V_{2}} u_{2}^{2} \phi_{3} \frac{\partial g_{23}}{\partial u_{1}}-\frac{V_{1}}{V_{2}} \phi_{2} \phi_{3}^{2} \frac{\partial g_{23}}{\partial \phi_{3}}
\end{aligned}
$$

$$
\frac{\Delta u_{2}^{\mathrm{m}}}{R T}=\ln \phi_{2}+\phi_{1}\left(1-\frac{V_{2}}{V_{1}}\right)+\phi_{3}\left(1-\frac{V_{2}}{V_{3}}\right)+\left(g_{12} \frac{V_{2}}{V_{1}} \phi_{1}+g_{23} \phi_{3}\right)\left(\phi_{1}+\phi_{3}\right)
$$




$$
\begin{aligned}
& -\frac{V_{2}}{V_{1}} u_{1}^{2} \phi_{2} \frac{\partial g_{12}}{\partial u_{2}}-g_{13} \frac{V_{2}}{V_{1}} \phi_{1} \phi_{3}+\frac{V_{2}}{V_{1}} u_{1}^{2} \phi_{3} \frac{\partial g_{13}}{\partial u_{2}} \\
& -\frac{V_{2}}{V_{1}} \phi_{1} \phi_{3}^{2} \frac{\partial g_{13}}{\partial \phi_{3}}-u_{1} u_{2} \phi_{3} \frac{\partial g_{23}}{\partial u_{1}}-\phi_{2} \phi_{3}^{2} \frac{\partial g_{23}}{\partial \phi_{3}}
\end{aligned}
$$

According to the Flory-Huggins thermodynamics, the Gibbs free energy of mixing for the binary phase is given by eqn. (8) (where $x_{t}$ is the mole fraction of component $i$ in the binary liquid)

$\frac{\Delta G_{\mathrm{m}}}{R T}=x_{1} \ln v_{1}+x_{2} \ln v_{2}+g_{12}\left(v_{2}\right) x_{1} v_{2}$

Differentiation with respect to $x_{1}$ and $x_{2}$ yields eqns. (9) and (10)

$\frac{\Delta \mu_{1}^{o}}{R T}=\ln v_{1}+\left(1-\frac{V_{1}}{V_{2}}\right) v_{2}+g_{12} v_{2}^{2}-v_{1} v_{2}^{2} \frac{\partial g_{12}}{\partial v_{2}}$

$\frac{\Delta \mu_{2}^{\circ}}{R T}=\ln v_{2}+\left(1-\frac{V_{2}}{V_{1}}\right) v_{1}+\frac{V_{2}}{V_{1}} g_{12} v_{1}^{2}+\frac{V_{2}}{V_{1}} v_{2} v_{1}^{2} \frac{\partial g_{12}}{\partial v_{2}}$

Assuming $V_{1} / V_{3} \approx V_{2} / V_{3} \approx 0$ and $V_{1} / V_{2}=l$, substitution of eqns. (6), (7), (9) and (10) in eqns. (3) and (4) and elimination of $\Pi$ gives

$$
\begin{aligned}
\ln \left(\frac{\phi_{1}}{\phi_{2}}\right)-\ln \left(\frac{v_{1}}{v_{2}}\right)= & (l-1) \ln \frac{\phi_{2}}{v_{2}}-g_{12}\left(u_{2}\right)\left[\phi_{2}-\phi_{1}\right]-g_{12}\left(v_{2}\right)\left[v_{1}-v_{2}\right] \\
& -\phi_{3}\left(g_{13}-l g_{23}\right)+u_{1} \phi_{2} \frac{\partial g_{12}}{\partial u_{2}}-v_{1} v_{2} \frac{\partial g_{12}}{\partial v_{2}} \\
& +\phi_{3} u_{1} \frac{\partial g_{13}}{\partial u_{2}}-\frac{V_{1}}{V_{2}} u_{2} \phi_{3} \frac{\partial g_{23}}{\partial u_{1}}
\end{aligned}
$$

Krigbaum [9] used a different coefficient for expressing the preferential sorption, namely the composition ratio $C R\left(C R \equiv \phi_{1} / \phi_{2}\left(u_{1} / v_{2}\right)^{-1}\right.$ and $\ln C R=$ $\left.\ln \left(\phi_{1} / \phi_{2}\right)-\ln \left(v_{1} / v_{2}\right)\right)$. The preferential sorption coefficient $\epsilon$ and the composition ratio $C R$ are directly related to each other,

$\epsilon=\frac{(C R-1) v_{1} v_{2}}{\left[1+(C R-1) v_{1}\right]}$

The left hand side of eqn. (11) is equal to the logarithm of the composition ratio. One can see from eqn. (11) that this expression for the preferential sorption does not contain derivatives of $g_{13}$ and $g_{23}$ with respect to $\phi_{3}$ anymore, while in equations for the osmotic pressure these derivatives are present. 
If the interaction parameters are assumed to be concentration independent, eqn. (11) reduces to the same equations as have been derived by Scott [10] for systems with $l=1$ and by Krigbaum [9] for systems with $l \neq 1$.

From eqn. (11) the preferential sorption can be calculated numerically if the interaction parameters and their partial derivatives, the ratio of the molar volumes $l$ and the volume fraction of polymer $\phi_{3}$ (or the overall sorption) are known.

Evaluation of the binary interaction parameter $g_{12}$

In a recent article [4] it was shown that, if data on excess free energy of mixing are available $g_{12}\left(v_{2}\right)$ (or $g_{12}\left(u_{2}\right)$ ) can be calculated according to eqn.

$g_{12}=\frac{1}{x_{1} v_{2}}\left[x_{1} \ln \frac{x_{1}}{v_{1}}+x_{2} \ln \frac{x_{2}}{v_{2}}+\frac{\Delta G^{\mathbf{E}}}{R T}\right]$

This method of calculating $g_{12}$ values has also been used by others $[11,12]$.

For many liquid mixtures the thermodynamic excess functions are available. The $g_{12}$ function for ethanol-water has been calculated from literature data on $\Delta G^{E}$ taken from Westmeier [13]. This function, written as a fourth grade polynomial relation [4], is given by eqn. (14)

$g_{12}\left(v_{2}\right)=0.9820-1.3483 v_{2}+4.15 v_{2}^{2}-3.3116 v_{2}^{3}+0.8897 v_{2}^{4}$

For the liquid mixture in the polymer, $v_{2}$ has to be replaced by $u_{2}$. This $g_{12}\left(u_{2}\right)$ function is assumed to be independent of the polymer concentration. The derivatives of $g_{12}$ to $v_{2}$ and $u_{2}$ can easily be obtained from eqn. (14).

Evaluation of the binary parameters $g_{13}$ and $g_{23}$

Interaction parameters between a polymer and a nonsolvent can be determined experimentally by equilibrium swelling measurements, as has been described previously [4]. For the system studied the swelling measurements and interaction parameters are given in Table 1.

The parameters given in the last column of Table 1 are binary parameters. In order to consider second order or ternary effects the $g_{13}$ and $g_{23}$ parameters are assumed to be concentration dependent, i.e., $g_{13}$ and $g_{23}$ are functions of $u_{i}(i=1,2)$ and $\phi_{3}$. We will use such a mathematical expression for these parameters that if the concentration $u_{2}$ in the polymer increases, $g_{13}$ will increase, and if $u_{1}$ increases, $g_{23}$ will increase. Furthermore, if the polymer concentration increases both $g_{13}$ and $g_{23}$ will increase.

$g_{13}=g_{13}\left(u_{2} \rightarrow 0\right)+a u_{2}+b\left[\phi_{3}-\phi_{3}\left(u_{2} \rightarrow 0\right)\right]$
$g_{23}=g_{23}\left(u_{1} \rightarrow 0\right)+c u_{1}+d\left[\phi_{3}-\phi_{3}\left(u_{1} \rightarrow 0\right)\right]$

For the limiting cases $u_{2} \rightarrow 0$ and $u_{1} \rightarrow 0$ eqns. (15) and (16) reduce to $g_{13}=$ $g_{13}\left(u_{2} \rightarrow 0\right)=\chi_{13}$ and $g_{23}=g_{23}\left(u_{1} \rightarrow 0\right)=\chi_{23}$. The values of the constants $g_{13}\left(u_{2} \rightarrow 0\right), g_{23}\left(u_{1} \rightarrow 0\right), \phi_{3}\left(u_{2} \rightarrow 0\right)$ and $\phi_{3}\left(u_{1} \rightarrow 0\right)$ have been given in Table 1. 
TABLE 1

Solubility and interaction parameters of water (component 1) and ethanol (component 2) in the polymers (3) cellulose acetate (CA), polyacrylonitrile (PAN) and polysulfone (PSf)

\begin{tabular}{lllll}
\hline Polymer & Penetrant & $\begin{array}{l}\text { Solubility } \\
\text { (g penetrant/100 g dry polymer) }\end{array}$ & $\begin{array}{l}\text { Volume fraction } \\
\text { of polymer }^{\mathrm{a}}\end{array}$ & $\chi^{\mathrm{b}}$ \\
\hline CA & water & 14.3 & 0.84 & 1.4 \\
PAN & water & 8.9 & 0.91 & 1.8 \\
PSf & water & 0.1 & 0.999 & 5.9 \\
CA & ethanol & 21.5 & 074 & 1.1 \\
PAN & ethanol & 0.1 & 0.994 & 4.2 \\
PSf & ethanol & 2.3 & 0.96 & 2.5 \\
\hline
\end{tabular}

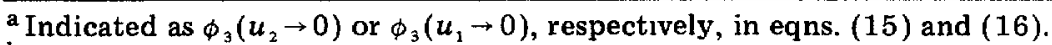

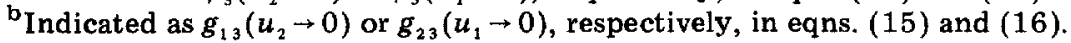

The molecular interpretation of the magnitudes of the constants $a, b, c$ and $d$ is left for future study. These coefficients can be chosen in such a way as to improve the agreement between theory and experiment, as we will see later on

\section{Experimental}

\section{Materials}

Cellulose acetate (E 398-3) was obtained from Eastman Chemicals, polysulfone (P 3500) from Union Carbide and polyacrylonitrile ( $T$ 75) from DuPont. The solvents used were of analytical grade.

\section{Membrane preparation}

Polymer solutions were prepared by dissolving the polymer in a suitable solvent. The membranes were prepared by casting the polymer solution upon a glass plate and allowing the solvent to evaporate in a nitrogen atmosphere. The membranes used were completely transparant except for that consisting of polyacrylonitrile.

\section{Swelling measurements}

Dried strips of polymeric membrane (about $0.3 \mathrm{~g}$ ) were immersed in different conical flasks containing water-ethanol mixtures of different compositions. The flasks were placed in a thermostat bath at $20^{\circ} \mathrm{C}$. After 24 hours the strips were removed, pressed between a tissue paper and weighed in a closed flask. This procedure was continued until no further weight increase was observed. The solubility has been expressed as a relative weight increase ( $\mathrm{g}$ penetrant/100 $\mathrm{g}$ dry polymer).

\section{Pervaporation}

The pervaporation experiments were carried out as described previously [14]. Vacuum at the downstream side was maintained at a pressure of $13.3 \mathrm{~Pa}$ 
$(0.1 \mathrm{mmHg})$ by a Crompton-Parkinson vacuum pump. The pressure was measured-by an Edwards piranhi gauge. The experiments were carried out for eight hours. A product sample was taken every hour and generally steady-state conditions were reached in about three hours. The thickness of the homogeneous membranes was about $20 \mu \mathrm{m}$. The temperature of the liquid feed mixture was $20^{\circ} \mathrm{C}$.

\section{Product analysis}

Analysis of binary ethanol-water mixtures was performed on a Varian model 3700 gaschromatograph fitted with a chromosorb $60 / 80$ column and equipped with a thermal conductivity detector. For low ethanol concentrations $(0-5 \%)$ a flame ionization detector was used.

\section{Analysis of the binary liquid mixtures inside the polymeric membrane}

The composition of the liquid mixture in the polymeric membrane was determined by a distillation technique as described by Patat [15]. The experiments were carried out with the apparatus given in Fig. 1. The apparatus was flushed thoroughly with nitrogen before the experiments were started. The polymeric membrane was immersed in a conical flask containing the binary ethanol-water mixture. After sorption equilibrium, which can be controlled by repeated weighing, the membrane sample was pressed between tissue paper and immediately put in tube 1 . The closed tube 1 was cooled with liquid nitrogen and installed in the apparatus. The system was brought to a pressure of about $1.3 \mathrm{~Pa}(0.01 \mathrm{mmHg})$ while tube 1 was still cooled. After about 5-10 minutes, valve 5 was closed, tube 2 was cooled with liquid nitrogen (the level up to which cooling is performed is indicated by the dashed line) and tube 1 was heated with boiling water. Within 10-30 seconds, the liquid inside the membrane started to boil and the vapour was condensed in tube 2 . After about 10-15 minutes the experiment was stopped because no more liquid could be removed from the membrane. This was checked in two ways: by

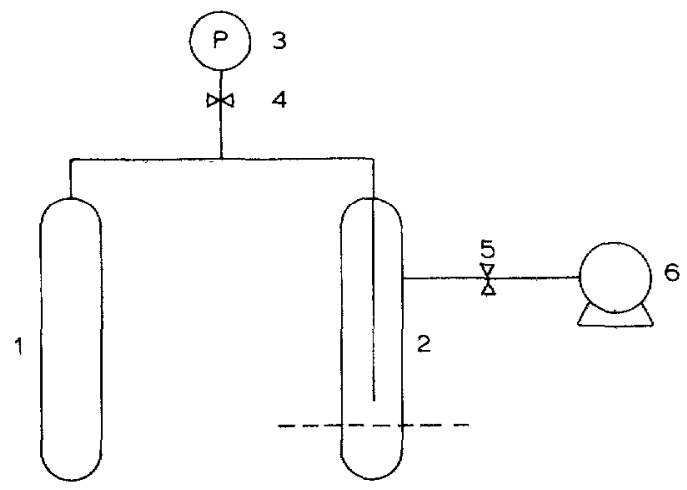

Fig. 1. Apparatus to determine the composition of the liquid mixture inside the polymer; 1,2 - collecting tubes, 3 - piranhi gauge, 4,5-valves, 6 -vacuum pump. 
following the pressure during the experiment and by performing experiments for longer periods of time. The amount of liquid inside the membrane could be determined by simply weighing, while the composition was determined by gas chromatography.

The experimental error depends on the amount of liquid sorbed by the membrane. The maximum amount of liquid sorbed in a PSf membrane is about $3 \%$, while for CA membranes up to $25 \%$ was sorbed. The error in the mass balance is about $5 \%$ for polysulfone systems.

\section{Results and discussion}

The experimental total sorption values of ethanol-water mixtures in cellulose acetate, polysulfone and polyacrylonitrile are given in Fig. 2. Figure 2 demonstrates clearly the difference in thermodynamic behaviour between the different polymers and ethanol-water mixtures; low swelling values in PSf and PAN and much higher values in CA are observed. PSf and PAN show opposite behaviour, hardly any water sorption in PSf while PAN shows hardly sorption for ethanol. The solubility of ethanol-water mixtures in CA passes through a maximum at about $65 \%$ ethanol in the feed.

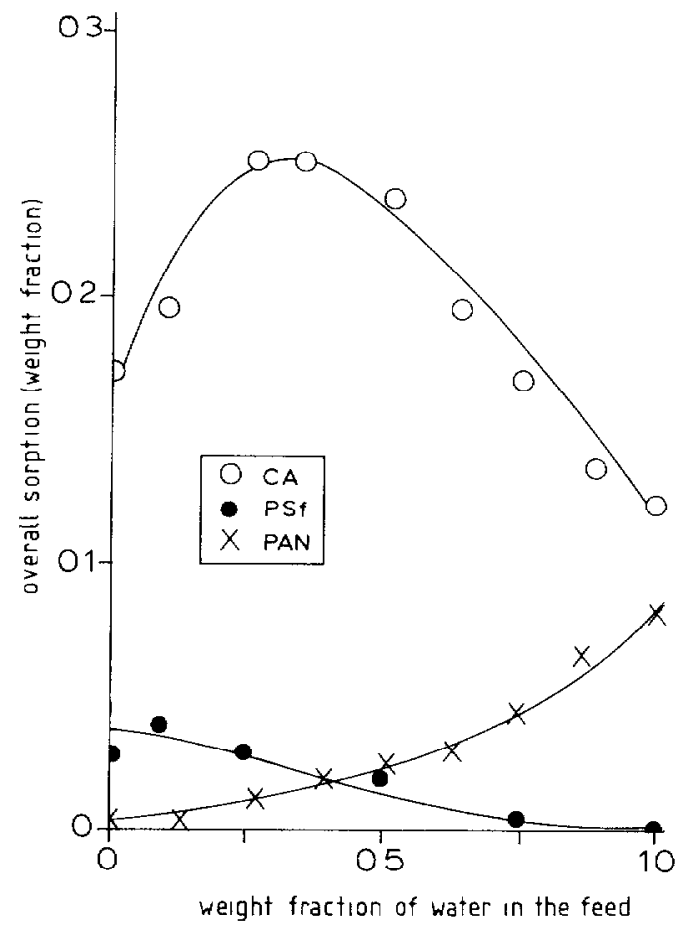

Fig. 2. Total sorption as a function of the water content in the water-ethanol liquid feed mixture for different polymers at $20^{\circ} \mathrm{C}$. 
Values for the preferential sorption have been determined experimentally and theoretically. The theoretical values can be calculated from eqn. (11). Equation (11) shows that preferential sorption depends on the difference in molar volumes of the two penetrants, the affinity of both components towards the polymer and the mutual interaction between the two penetrants.

The effect of the difference in molar volume has its origin in the combinatorial entropy, and this effect upon the preferential sorption increases if the difference in molar volume increases, and if the polymer concentration increases. The component with the smaller molar volume will be sorbed preferentially. For water-ethanol the ratio of the molar volumes $l\left(=V_{1} / V_{2}\right)$ is 0.31 , which means that water will be sorbed preferentially.

Positive values of the term containing the interaction parameters with respect to the polymer $\left(g_{23}-g_{13}\right)$ will favour the preferential sorption of component 1; this effect also depends on the polymer concentration. For the systems studied this value is negative. Hence, this term contributes to selectivity towards component 2 (ethanol).

The influence of $g_{12}$ on the preferential sorption depends on the concentration in the binary liquid phase and on the sign of the $g_{12}$ interaction parameter. In the case of water-ethanol the interaction parameter $g_{12}$ has a positive value over the entire composition range. This implies that the term $g_{12}\left(v_{2}-v_{1}\right)$ has a positive effect on the preferential sorption of water for high ethanol feed concentrations $\left(v_{2}>v_{1}\right)$, while it has a negative effect for high water feed concentrations $\left(v_{1}>v_{2}\right)$. The same applies for $g_{12}\left(\phi_{1}-\phi_{2}\right)$, but the effect of this term is much smaller because $\phi_{1}$ and $\phi_{2}$ are smaller for the systems studied compared to $v_{1}$ and $v_{2}$.

The influence of the derivatives of $g_{13}$ and $g_{23}$ with respect to $u_{2}$ and $u_{1}$, respectively, depends on the magnitudes of the constants $a$ and $c$ (see eqns. 15 and 16). The term containing the derivative $\partial g_{12} / \partial v_{2}$ has a positive effect on the preferential sorption of water, while the term with $\partial g_{12} / \partial u_{2}$ has a negative effect, the latter being smaller than the former.

When neglecting the concentration dependency of the interaction parameters, it can be deduced from eqn. (11) that for the limiting case $\phi_{3} \rightarrow 1$, the liquid mixture inside the polymer consists almost exclusively of the component with the smaller molar volume.

Some numerical examples will demonstrate the influence of the different factors (difference in molar volume, difference in affinity towards the polymer and the mutual interaction between ethanol and water) on the preferential sorption applied to ethanol-water mixtures. Figure 3 gives the preferential sorption of ethanol-water for different polymer concentrations assuming equal and constant polymer-nonsolvent parameters $\left(\chi_{13}=\chi_{23}=1.0\right)$. For $g_{12}$ (water-ethanol) eqn. (14) has been used. It is obvious that the effect of the difference in molar volume on the preferential sorption of water becomes stronger if the polymer concentration increases.

Figure 4 is an example of an opposite effect. The smaller molar volume of water, which favours the preferential sorption of water, is opposed by the 


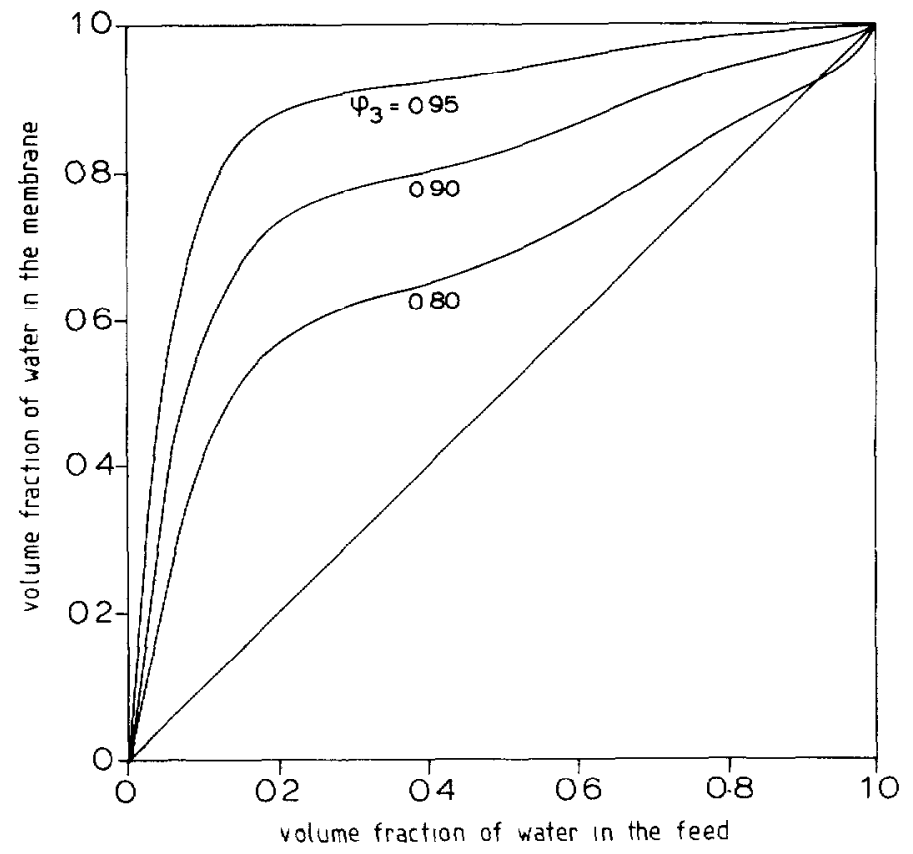

Fig. 3. Preferential sorption in a ternary system water-ethanol-polymer for different polymer concentrations $\left(\phi_{3}=0.95 ; \phi_{3}=0.9 ; \phi_{3}=0.8\right)$. Other parameters: $\chi_{13}=\chi_{23}=1.0$; $g_{12}=g_{12}\left(u_{2}\right)$ (see eqn. 14$) ; l=0.31$.

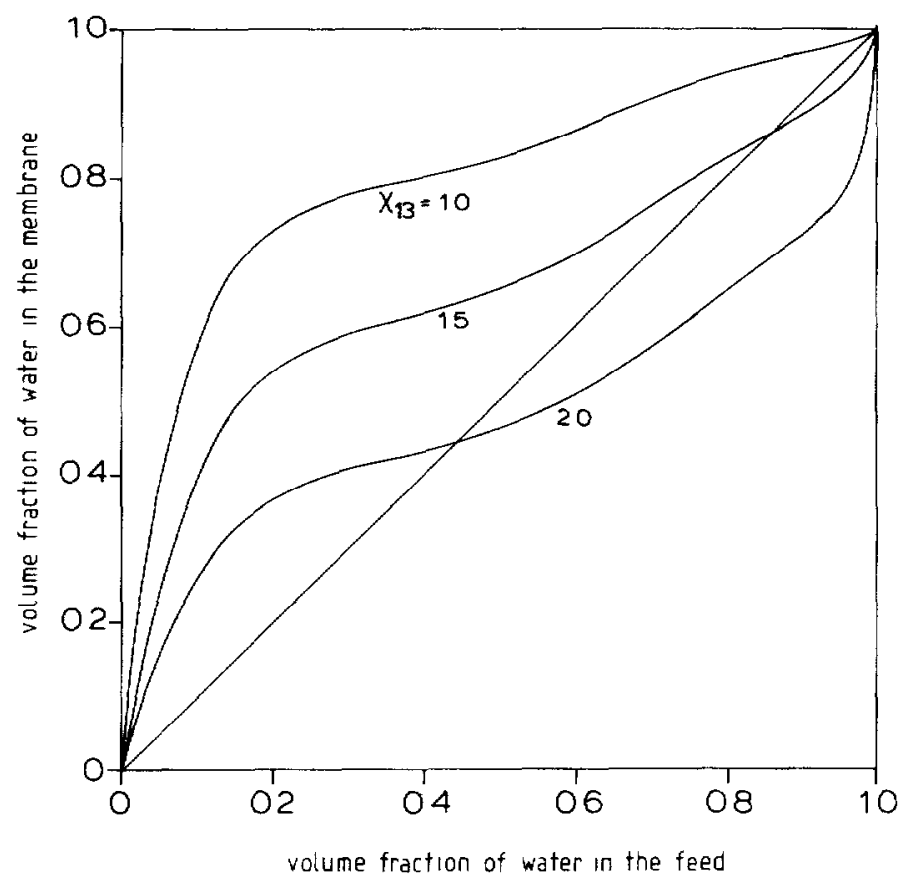

Fig. 4. Preferential sorption in a ternary system water-ethanol-polymer for different values of $\chi_{13}\left(\chi_{13}=10 ; \chi_{13}=1.5 ; \chi_{13}=2.0\right)$. Other parameters: $\chi_{23}=1.0 ; g_{12}=g_{12}\left(u_{2}\right)$ (see eqn. 14 ) $; l=0.31 ; \phi_{3}=0.90$ 
small mutual affinity of water and polymer. When the $\chi_{13}$ parameter increases, keeping $\chi_{23}$ constant, the preferential sorption of water decreases and even an inversion in preferential sorption can be observed. Furthermore the lower curve $\left(\chi_{13}=2.0\right)$ of Fig. 4 demonstrates clearly the influence of the $g_{12}$ parameter on the preferential sorption: $g_{12}$ is positive over the entire composition range (see eqn. 14), and the contribution to the preferential sorption is positive for low concentrations of water in the feed, while it has a negative effect for high concentrations of water in the feed.

In case of polysulfone and polyacrylonitrile as membrane materials, the polymer concentration, $\phi_{3}$, is quite high (see Fig. 2). For the system waterethanol-polyacrylonitrile we expect a large preferential sorption for water, because the component with the smaller molar volume (water) has also a larger affinity towards the polymer (see Table 1 and Fig. 2). For the system waterethanol-polysulfone, the preferential sorption for water due to the effect of the smaller molar volume is counteracted by the very small affinity of water to polysulfone. In the next section we will see how the experimental and theoretical data agree.

The system water-ethanol-cellulose acetate (sorption results)

In Fig. 5 the theoretical values for the preferential sorption calculated according to eqn. (11) with constant interaction parameters $\left(g_{i j}=\chi_{2 j}\right)$ and with concentration dependent interaction parameters, together with the experimental values are given as a function of the water concentration in the feed. The experimental values show that water is sorbed preferentially over the entire composition range. The preferential sorption increases with decreasing water concentration in the feed mixture.

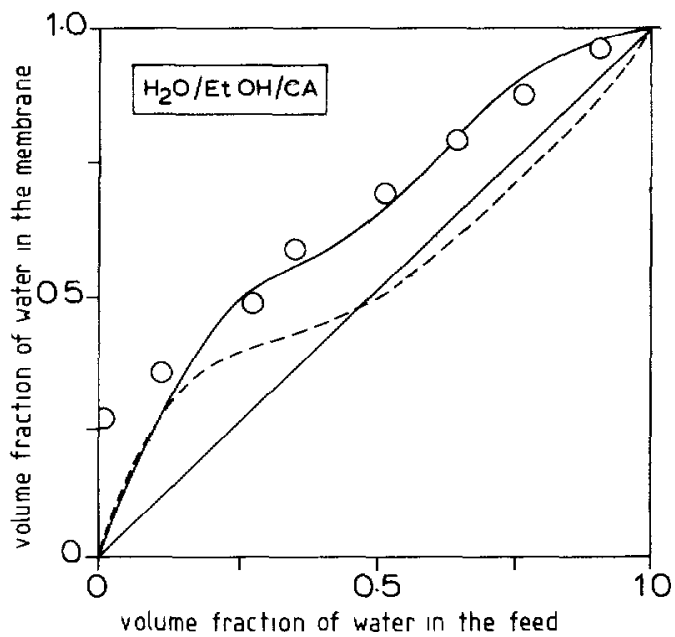

Fig. 5. Experimental values $(O)$ for the preferential sorption (expressed as volume fraction of water inside the polymer) and calculated values using concentration dependent (full curve) and constant (dotted curve) interaction parameters as a function of the volume fraction of water in the liquid feed, for the system water-ethanol-cellulose acetate. 
From the calculated values when using constant polymer-nonsolvent interaction parameters, an inversion of the preferential sorption can be observed which is not in agreement with the experimental observations. To improve agreement between the experimental and theoretical values, concentration dependent $g_{13}$ and $g_{23}$ parameters have been used. By investigating the effect of the constants $a, b, c$ and $d$ (see eqns. 15 and 16) on the preferen. tial sorption, it became clear that a reasonable agreement between theory and experiment was obtained when the coefficient $a$ has a higher value than $c$. The concentration dependence of $g_{13}$ and $g_{23}$ is given in Fig. 6 .

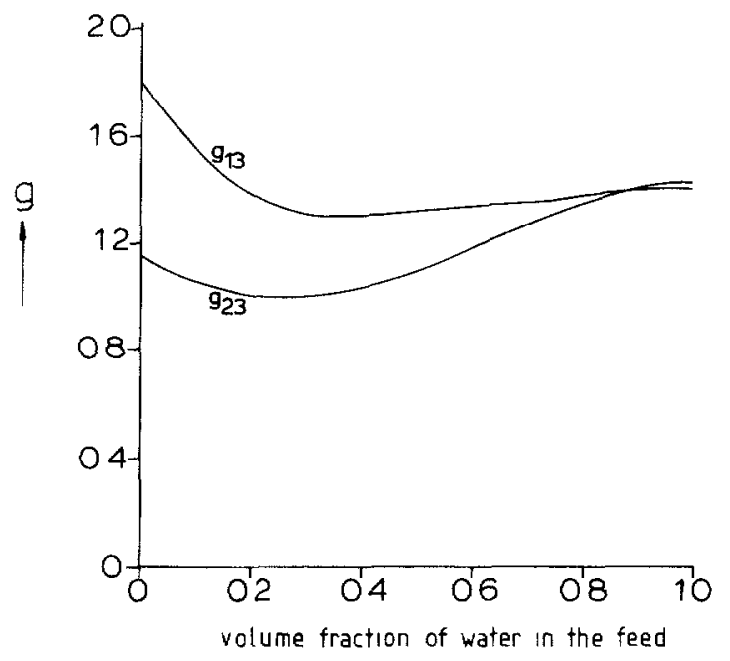

Fig. 6. Interaction parameters $g_{13}$ and $g_{23}$ in the system water-ethanol-cellulose acetate as a function of the water concentration in the feed. Parameters used in eqns. (15) and (16) are $\cdot a=0.6 ; b=2.2 ; c=0.1 ; d=2.2$.

The influence of the $g_{13}$ and $g_{23}$ parameters on the preferential sorption is caused to a large extent by their derivatives and the choice of the coefficients $a$ and $c$. Hence $a>c$ results in $\partial g_{13} / \partial u_{2}>\partial g_{23} / \partial u_{1}$ which has a positive effect on the preferential sorption of water (see eqn. 11).

The system water-ethanol-polyacrylonitrile (sorption results)

The experimental and theoretical values for the preferential sorption are given in Fig. 7. The theoretical values have been calculated according to eqn. (11) using constant interaction parameters given in Table $1\left(g_{13}=\chi_{13}=1.8\right.$ and $g_{23}=\chi_{23}=4.2$ ).

For low water concentrations in the feed no experimental values could be obtained because the amount of sorbed liquid was too small (see Fig. 2). From Fig. 7 very high values for the preferential sorption of water can be observed. This behaviour could already be expected because of the smaller molar volume of water and the much higher affinity between water and poly- 
acrylonitrile in comparison with ethanol and polyacrylonitrile. The theoretical values hardly change when concentration dependent $g_{13}$ and $g_{23}$ parameters were used. Because of the very high fractional water content inside the membrane, the value of $g_{13}$ will not change very much $\left(u_{2} \rightarrow 0\right.$, see eqn. 15) while a higher value of $g_{23}$ has no influence on the preferential sorption.

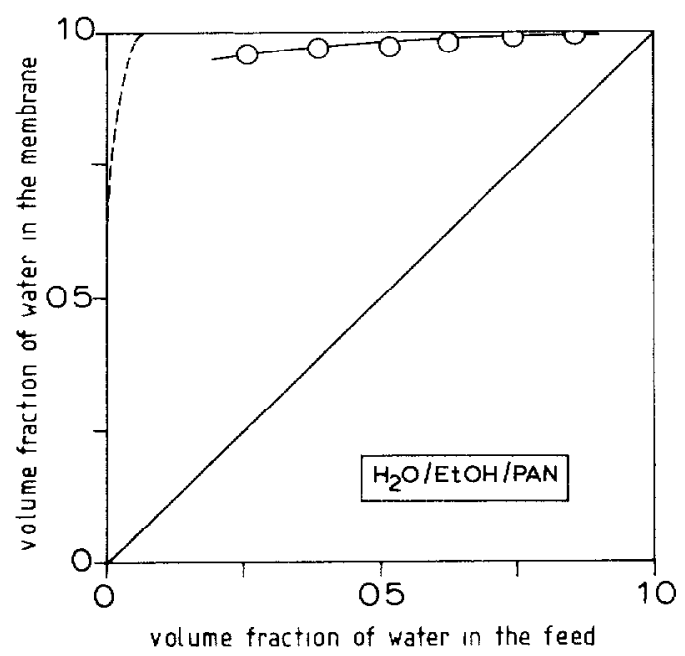

Fig. 7. Experimental $(0)$ and theoretical $(---)$ values for the preferential sorption in the system water-ethanol-polyacrylonitrile as a function of the volume fraction of water in the liquid feed.

The system water-ethanol-polysulfone (sorption results)

This system is a very interesting one because the effect of the difference in molar volumes, contributing to a large extent to the preferential sorption of water in the two other systems studied, is opposed here by the very small mutual affinity between water and polysulfone.

Because a very small amount of liquid is sorbed by polysulfone, especially at high water concentrations in the feed, only one experimental value has been obtained at a high ethanol feed concentration. The theoretical and experimental results are given in Table 2.

\section{TABLE 2}

Theoretical and experimental value of preferential sorption in the system water-ethanolpolysulfone

\begin{tabular}{lll}
\hline & $\begin{array}{l}\text { Water in feed }\left(v_{1}\right) \\
\text { (weight fraction) }\end{array}$ & $\begin{array}{l}\text { Water in membrane }\left(u_{1}\right) \\
\text { (weight fraction) }\end{array}$ \\
\hline $\begin{array}{ll}\text { Experimental } \\
\text { Theoretical }\end{array}$ & 0.11 & 0.23 \\
& 0.11 & 0.28 \\
\hline
\end{tabular}

Parameters used in eqns. (15) and (16) are: $a=0.2 ; b=65.0 ; c=1.2 ; d=65.0$. 
Using concentration independent interaction parameters, the term $l_{\chi_{23}}-\chi_{13}(=-5.1)$ which is very large, dominates completely and predicts a preferential sorption of component 2 (ethanol). By taking the interaction parameters concentration dependent, preferential sorption of water can be calculated. However, the coefficients $a, b, c$ and $d$ (see eqns. 15 and 16) have completely different values as in the case of cellulose acetate. Although a reasonable agreement can be obtained between theory and experiment, the physical interpretation of the values for the coefficients $a, b, c$ and $d$ is still unexplained. Nevertheless, it is striking that even in hydrophobic polymers such as polysulfone, water is sorbed preferentially.

\section{Preferential sorption versus preferential permeation}

The main objective of this paper was to investigate preferential sorption in relation to selective transport in pervaporation. Successively, we will discuss the systems water-ethanol-cellulose acetate, water-ethanol-polyacrylonitrile and water-ethanol-polysulfone.

The system water-ethanol-cellulose acetate (sorption vs. permeability)

In Fig. 8 the experimental sorption values and the results for pervaporation are given as a function of the weight fraction of water in the feed mixture. It is obvious that both curves show practically the same behaviour. If these results are considered in terms of the solution-diffusion model, where the flux of a component through a membrane is a function of solubility, $S$, and

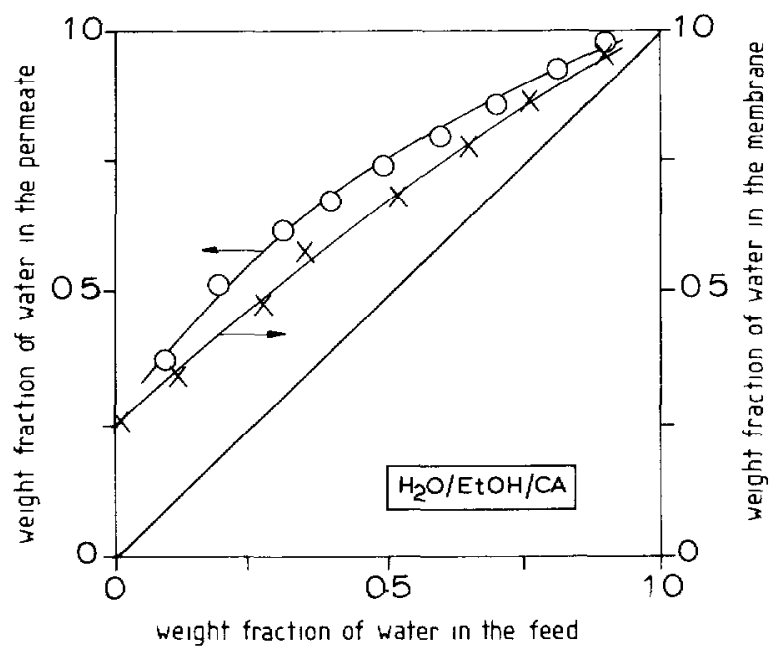

Fig. 8. Experimental values for preferential sorption (expressed as weight fraction of water inside the polymer) and for pervaporation (expressed as weight fraction of water in the permeate) for the system water-ethanol-cellulose acetate as a function of the weight fraction of water in the feed. 
diffusivity, $D$, it is clear that solubility contributes to a major part to selective transport

$\frac{J_{1}}{J_{2}}=\frac{S_{1}\left(c_{1}, c_{2}\right)}{S_{2}\left(c_{1}, c_{2}\right)} \quad \frac{D_{1}\left(c_{1}, c_{2}\right)}{D_{2}\left(c_{1}, c_{2}\right)}$

In the case of water and ethanol permeating through cellulose acetate, both the ratios $S_{1} / S_{2}$ and $D_{1} / D_{2}$ are larger than one. For water and ethanol the ratio $D_{1} / D_{2}$ is larger than one because of the difference in size of the molecules (see for instance Berens [16]). So in order to obtain very high selectivities the ratios $S_{1} / S_{2}$ and/or $D_{1} / D_{2}$ should be very large. For the system waterethanol-cellulose acetate the ratio $S_{1} / S_{2}$ is rather low and moderate selectivities are obtained, implying that the ratio $D_{1} / D_{2}$ will not be very large either.

The system water-ethanol-polyacrylonitrile (sorption vs. permeability)

For this system the experimental preferential sorption values and the results for pervaporation as a function of the weight fraction of water in the feed are given in Fig. 9. Again, both curves show the same behaviour.

Because the ratio $S_{1} / S_{2}$ is much larger here compared to that for cellulose acetate, very high selectivities could be expected and are indeed achieved. For this system also, the contribution of the preferential sorption to the selectivity in permeation turns out to be the leading factor.

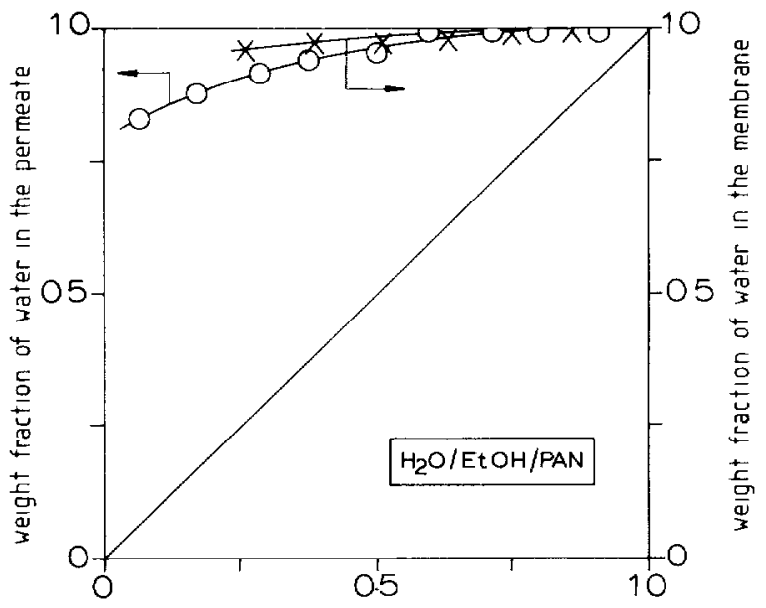

weight fraction of water in the feed

Fig. 9. Experimental values for preferential sorption and pervaporation for the system water-ethanol-polyacrylonitrile as a function of the weight fraction of water in the feed.

The system water-ethanol-polysulfone (sorption vs. permeability)

In a previous section it was shown that water is sorbed preferentially from an ethanol-water mixture in the strongly hydrophobic polymer polysulfone. Analogous to the other systems studied, a preferential permeability for water 
should be expected. In Fig. 10, the one experimental sorption value and a curve for pervaporation results are given as a function of the weight fraction of water in the liquid feed. As was found for the other two systems, preferential sorption and preferential permeation show parallel behaviour, but in contrast to the other two systems, the sorption selectivity is much lower than the selectivities found in the pervaporation process. We think that in this system the mobility of the ethanol molecules has been decreased because of the hydrophobic interactions between ethanol and polysulfone. As a consequence the ratio $D_{1} / D_{2}$ should be very large.

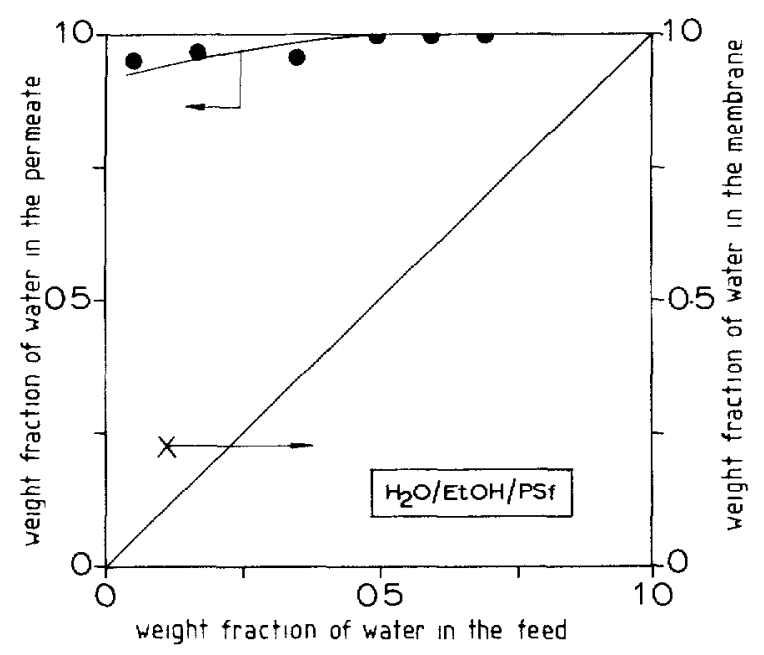

Fig. 10. Experimental values for preferential sorption (one point, $x$ ) and pervaporation for the system water-ethanol-polysulfone as a function of the weight fraction of water in the feed.

The system water-ethanol-polysulfone is a perfect example to demonstrate the presence of coupled transport. For pure water no sorption nor permeation can be observed, while in the presence of ethanol water is sorbed and transported preferentially. In several models $[3,17]$ it has been tried to predict selectivity and permeation rates from parameters obtained from singlecomponent experiments. The results presented here demonstrate clearly that it would be hardly possible to predict membrane characteristics for non-ideal mixtures like water-ethanol from single-component experiments only. Coupling occurs in the thermodynamic part ("solution") as well as in the kinetic part ("diffusion") of the solution-diffusion mechanism.

Another interesting aspect which can be deduced from our experiments is that the assumption of ideal sorption behaviour cannot be used for nonideal mixtures such as ethanol-water. This can be demonstrated clearly by the system water-ethanol-polysulfone (but also by the other two systems). Krewinghaus [18] assumed a linear relationship (ideal sorption) between the 
concentration of a compound in the membrane and the concentration of that component outside the membrane, according to eqn. (18)

$c_{\imath}=x_{i} c_{\imath}^{\mathbf{o}}$

where $c_{\imath}$ is the concentration of component $i$ in the membrane, $x_{\imath}$ is the mole fraction of component $i$ in the liquid feed mixture and $c_{l}^{\circ}$ is the solubility in the membrane for the pure component. Equation (18) cannot be used for the system water-ethanol-polysulfone, because for pure water there is no sorption in a polysulfone membrane while in the presence of ethanol, water is sorbed preferentially. In general, one can say that in cases where preferential sorption occurs eqn. (18) cannot be used.

\section{Conclusions}

Using Flory-Huggins thermodynamics, extended with concentration depen. dent interaction parameters, the agreement between theoretical and experimental values for the preferential sorption of low molecular weight components in polymeric membranes is reasonably well established. For the systems studied, preferential sorption of water from ethanol-water mixtures occurs, and this can be ascribed to the large difference in molar volume between water and ethanol. Comparison of the preferential sorption values and the results for preferential permeation show that preferential sorption of water contributes to a large extent to selective water transport. From the results presented here it can be predicted that high selectivities for water from ethanol-water mixtures can be expected when the total amount of liquid inside the polymer is small (in this case the permeation rates will be low, however), and when the affinity between water and polymer is larger than that between ethanol and polymer.

In terms of the solution-diffusion model the conclusion is justified that the component that is sorbed preferentially will also permeate preferentially. This statement is in agreement with the observations of Aptel [5]. Furthermore it can be concluded that the assumption of ideal sorption cannot be used in general.

\section{Acknowledgement}

This paper is based upon work financially supported by the Ministeries van Economische Zaken en Onderwijs en Wetenschappen in the Netherlands.

\section{List of symbols}

a activity

$c \quad$ concentration inside the membrane

$c^{\circ} \quad$ solubility of the pure component in the membrane

CR composition ratio 


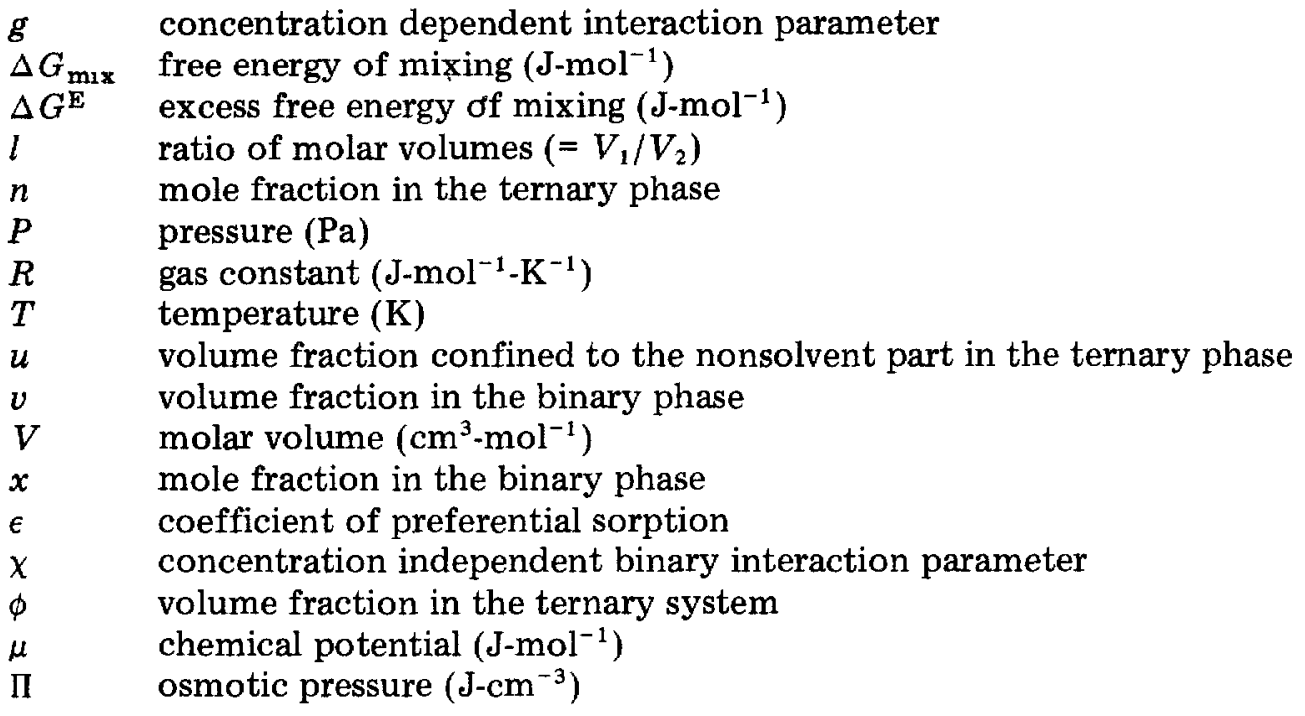

$\begin{array}{ll}\text { Indices } & \\ 1 & \text { water } \\ 2 & \text { ethanol } \\ 3 & \text { polymer } \\ i & \text { component } i \\ \mathrm{~m} & \text { membrane }\end{array}$

\section{References}

1 R.C Binning, R.J. Lee, J.F. Jennings and E.C Martin, Separation of liquid mixtures, Ind. Eng. Chem., 53 (1961) 45.

2 D.R. Paul and J.D. Paciotti, Driving force for hydraulic and pervaporative transport in homogeneous membranes, J. Polym. Sci. A-2, 13 (1975) 1201

3 C.H. Lee, Theory of reverse osmosis and some other membrane permeation operations, J. Appl. Polym. Sci, 19 (1975) 83.

4 M.H.V. Mulder and C.A. Smolders, On the mechanism of separation of ethanol/water mixtures by pervaporation. I. Calculation of concentration profiles, J. Membrane Sci., 17 (1984) 289.

5 P. Aptel, J Cuny, J. Jozefowicz, J Neel and B. Chaufer, Perméabilité sélective et solvatation préférentielle, Eur. Polym. J., 14 (1978) 595

6 P. Flory, Principles of Polymer Chemistry, Cornell University Press, Ithaca, New York, 1953.

7 A. Zivný and J. Pouchlý, Theoretıcal analysis of a binary solvent in a polymer phase. I. Occurrence and character of inversion in preferential sorption, J. Polym. Sci. A-2, 10 (1972) 1467.

8 J. Pouchly and A. Zivný, Correlation of data on preferential sorption using the modified Flory-Huggins equation, Makromol Chem, 183 (1982) 3019

9 W.R. Krigbaum and D K. Carpenter, Phase equilibria in polymer-liquid 1-liquid 2 systems, J Polym. Sci., 14 (1954) 241. 
10 R.L. Scott, The thermodynamics of high polymer solutions. IV. Phase equilibria in the lernary system: Polymer-liquid 1-liquid 2, J. Chem. Phys, 17 (1949) 268.

11 F.W. Altena and C.A. Smolders, Calculation of liquid-liquid phase separation in a ternary system of a polymer in a mixture of a solvent and a nonsolvent, Macromolecules, 15 (1982) 1491

12 T.M. Aminabhavi and P. Munk, Preferential adsorption onto polystyrene in mixed solvent systems, Macromolecules, 12 (1979) 607.

13 S. Westmeier, Excessenthalpie, Freie Excessenthalpie, Excessvolumen und Viskosität von ausgewählten binären flüssigen Mischungen, Teil $H$. Die Systeme Wasser-Methanol und Wasser- Äthanol. Chem. Tech., 28 (1976) 350

14 M.H V. Mulder, F. Kruitz and C.A. Smolders, Separation of isomeric xylenes by pervaporation through cellulose ester membranes, J. Membrane Sci., 11 (1982) 349.

15 F. Patat and G. Träxler, Lösungsgleichgewichte von Makromolekulen I Die Zusammensetzung der Gelphase bei Fäll- und Lósefraktionierung, Makromol. Chem., 33 (1959) 113 .

16 A.R. Berens and H.B. Hopfenberg, Vapor sorption methods in studies of the glassy state: Part 1, sorption isotherms and diffusivities, 10th Europhysics Conference on Macromolecular Physics, Noordwijkerhout, The Netherlands, 1980, p 72.

17 F.W. Greenlaw, R.A Shelden and E.V Thompson, Dependence of diffusive permeation rates on upstream and downstream pressures. II Two component permeant, $\mathrm{J}$ Membrane Sci., 2 (1977) 333.

18 A.B Krewinghaus, Solution and transport of organic liquids and vapors in structurally modified polyethylenes, Ph.D. Thesis, Massachusetts Institute of Technology, April 1966. 